\title{
PRODUCTS OF COMMUTATORS AND PRODUCTS OF SQUARES IN A FREE GROUP
}

\author{
LEO P. COMERFORD, JR. AND CHARLES C. EDMUNDS
}

\begin{abstract}
A classification of the ways in which an element of a free group can be expressed as a product of commutators or as a product of squares is given. This is then applied to some particular classes of elements. Finally, a question about expressing a commutator as a product of squares is addressed.
\end{abstract}

\section{INTRODUCTION}

Our first aim is to provide a characterization of the set of all solutions to the equations

$$
\begin{gathered}
{\left[x_{1}, y_{1}\right] \ldots\left[x_{g}, y_{g}\right]=U} \\
x_{1}^{2} \ldots x_{g}^{2}=U
\end{gathered}
$$

in a free group, where $U$ is a product of $g$ but not fewer commutators in (11) and $U$ is a product of $g$ but not fewer squares and is not a product of fewer than $g / 2$ commutators in (2). Our description of the set of solutions, which we give in $\$ 2$, is based on work of C. Edmunds [8]. It provides an algebraic version of a result obtained by M. Culler [7, Theorem 4.1] by topological methods. This characterization of solutions is somewhat more explicit than those given by L. Comerford and Edmunds [6] and by R. Grigorchuk and P. Kurchanov [9] for a larger class of equations, and seems easier to use to get descriptions of solutions to particular equations. We use our method to classify solutions to certain classes of equations in $\S 3$. In $\S 4$ we give another application, this time to expressions of commutators as products of squares in a free group.

\section{Products of Commutators, Products of Squares}

We begin by fixing some notation and terminology. Let $H$ be the free group on $A=\left\{a_{1}, \ldots, b_{1}, \ldots, c_{1}, \ldots\right\}$ and let $F$ be the free group on $X=\left\{x_{1}, \ldots, y_{1}, \ldots\right\}$. We call elements of $A$ constants, elements of $X$ variables, and elements of $X \cup X^{-1}$ letters. Length of elements of $H$ or $F$ relative to these generating sets is denoted by $|\cdot|$. For $W \in F$, we let $\operatorname{Var}(W)$ be the set of variables occurring in $W$ and let $|\operatorname{Var}(W)|$ be its cardinality.

We denote by $G^{\prime}$ the commutator subgroup of a group $G$, and by $2 G$ the subgroup generated by squares of elements of $G$. For $U \in G^{\prime}$, we let genus ${ }^{+}(U)$ be the minimal number of commutators of which $U$ is a product, and for $U \in 2 G$, we let genus ${ }^{-}(U)$ be the minimal number of squares of which $U$ is a product. We set genus ${ }^{+}(1)=$

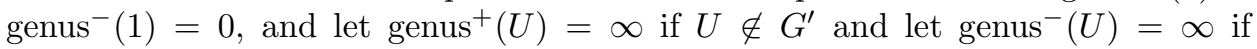
$U \notin 2 G$.

1991 Mathematics Subject Classification. Primary 20E05; Secondary 20 F10. 
Since $G^{\prime} \subseteq 2 G$ and since there are automorphisms of the free group on $x, y, z$ sending $x^{2}[y, z]$ and $x^{2} y^{2} z^{2}$ to one another, it follows that for $U \in G^{\prime}$, genus ${ }^{-}(U) \leq$ 2 genus $^{+}(U)+1$. There is, however, no general upper bound on genus ${ }^{+}(U)$ in terms of genus $^{-}(U)$ for $U \in G^{\prime}$. For instance, if $U=\left(\left[a_{1}, b_{1}\right] \ldots\left[a_{g}, b_{g}\right]\right)^{2}$, genus ${ }^{-}(U)=1$ but genus $^{+}(U)=2 g$ (cf. [5]).

An element $W$ of $F$ is called quadratic if each variable that occurs in $W$ occurs exactly twice, with exponents +1 or -1 . We call a quadratic word $W$ orientable if $W \in F^{\prime}$, and nonorientable otherwise. Note that every quadratic word is an element of $2 F$. A quadratic word $W$ is irredundant if there is no pair of distinct, noninverse letters $x, y$ which appear in $W$ only in subwords $(x y)^{ \pm 1}$.

With any quadratic word $W$ we may associate a closed surface $S_{W}$ by writing $W$ around the boundary of a disk and identifying edges labeled by the same variable, respecting orientation. Note that the orientability of $S_{W}$ is the same as that of $W$. We define the Euler characteristic of $W, \chi(W)$, to be that of $S_{W}$. By classical results, $\chi(W)=2-2$ genus $^{+}(W)$ if $W$ is orientable and $\chi(W)=2-$ genus $^{-}(W)$ if $W$ is nonorientable. Also, if $\alpha$ is an automorphism of $F$ and both $W$ and $W \alpha$ are quadratic, $\chi(W)=\chi(W \alpha)$. If $W$ is an orientable quadratic word with genus ${ }^{+}(W)=g$, there is an automorphism $\alpha$ of $F$ with $W \alpha=\left[x_{1}, y_{1}\right] \ldots\left[x_{g}, y_{g}\right]$, and if $W$ is a nonorientable quadratic word with genus ${ }^{-}(W)=g$, there is an automorphism $\alpha$ of $F$ with $W \alpha=x_{1}^{2} \ldots x_{g}^{2}$.

A solution to an equation

$$
W\left(x_{1}, \ldots\right)=U\left(a_{1}, \ldots\right)
$$

is a homomorphism $\phi: F \rightarrow H$ such that $W \phi=U$. A solution $\phi$ to (3) is called cancellation-free of $x \phi \neq 1$ for each variable $x$ occuring in $W$ and if $W\left(x_{1} \phi, \ldots\right) \equiv$ $U\left(a_{1}, \ldots\right)$, where $\equiv$ denotes equality in the free semigroup on $A \cup A^{-1}$.

Finally, we need to say something about stabilizers of elements of $F$. For $W \in F$, we define $F_{W}$ to be the subgroup of $F$ generated by $\operatorname{Var}(W)$, we let $\operatorname{Stab}_{F}(W)=\{\alpha \in \operatorname{Aut}(F): W \alpha=W\}$, and we let $\operatorname{Stab}_{F_{W}}=\left\{\alpha \in \operatorname{Aut}\left(F_{W}\right):\right.$ $W \alpha=W\}$. From a result of G. Rosenberger [16. Theorem 2.2], it follows that if $W=\left[x_{1}, y_{1}\right] \ldots\left[x_{g}, y_{g}\right]$ or $W=x_{1}^{2} \ldots x_{g}^{2}$ and if $\alpha \in \operatorname{Stab}_{F}(W)$, then $\alpha$ maps $F_{W}$ onto $F_{W}$. Thus, for these values of $W$, if $\alpha \in \operatorname{Aut}(F)$ and $W \alpha=W$, the restriction of $\alpha$ to $F_{W}$ is an element of $\operatorname{Aut}\left(F_{W}\right)$, so we need not distinguish between the stabilizers of $W$ in $F$ and in $F_{W}$, and we denote both by $\operatorname{Stab}(W)$.

Note that if $\phi$ is a solution to (3) and $\sigma \in \operatorname{Stab}_{F}(W)$, then $\sigma \phi$ is also a solution to (3). We say that two solutions $\phi_{1}$ and $\phi_{2}$ to (3) are in the same stabilizer class if $\phi_{2}=\sigma \phi_{1}$ for some $\sigma \in \operatorname{Stab}_{F}(W)$.

As general references, and for unexplained notation or terminology, we refer the reader to the books of $\mathrm{R}$. Lyndon and P. Schupp [13] and of W. Massey [15].

We are now ready to state our classification results, for the orientable and the nonorientable cases.

Theorem 1. Let $U$ be a nontrivial element of the free group $H$.

(a) If genus $^{+}(U)=g<\infty$, and $\phi$ is a solution to

$$
\left[x_{1}, y_{1}\right] \ldots\left[x_{g}, y_{g}\right]=U
$$

there is an irredundant orientable quadratic word $W \in F$ with genus ${ }^{+}(W)=g$ and a cancellation-free solution $\psi$ to $W=U$ such that for any automorphism 
$\gamma_{W}$ of $F$ with $W \gamma_{W}=\left[x_{1}, y_{1}\right] \ldots\left[x_{g}, y_{g}\right]$, there is an $\alpha \in \operatorname{Stab}\left(\left[x_{1}, y_{1}\right] \ldots\left[x_{g}, y_{g}\right]\right)$ such that $\phi=\alpha \gamma_{W}^{-1} \psi$.

(b) If genus $^{-}(U)=g<\infty$ genus $^{+}(U) \geq g / 2$, and $\phi$ is a solution to

$$
x_{1}^{2} \ldots x_{g}^{2}=U
$$

then there is an irredundant nonorientable quadratic word $W \in F$ with genus ${ }^{-}(W)=$ $g$ and a cancellation-free solution $\psi$ to $W=U$ such that for any automorphism $\gamma_{W}$ of $F$ with $W \gamma_{W}=x_{1}^{2} \ldots x_{g}^{2}$, there is an $\alpha \in \operatorname{Stab}\left(x_{1}^{2} \ldots x_{g}^{2}\right)$ such that $\phi=\alpha \gamma_{W}^{-1} \psi$.

We note that in this theorem we may assume, by composing with an inner automorphism if necessary, that $U$ is cyclically reduced.

In viewing $U$ as an image of a quadratic word $W$, it is convenient to take $U$ and $W$ to be cyclic words, that is, words written around a circle. If $U$ is an image of $W$ under a map $\psi$ as ordinary words, this remains the case if we view $U$ and $W$ as cyclic words. Our goal is to express $U$ as a cancellation-free image of a quadratic word $W$ under a map $\psi$. If this is the case with $U$ and $W$ cyclic words, we may be required to split a variable in $W$ to obtain such a representation as ordinary words. For example, $U=a^{-1} c^{-1} a b c b^{-1}$ is a cancellation-free image of $W=x^{-1} y^{-1} x y$ under $\psi: x \mapsto a b, y \mapsto c$ as cyclic words, but $U$ is not a cancellation-free image of $W$ as ordinary words. Since the ordinary word $U$ begins within the image of $x^{-1}$, we replace $x$ by $x_{1} x_{2}$ and define $\left(x_{1}\right) \psi=a$ and $\left(x_{2}\right) \psi=b$. We now find that as ordinary words, $U$ is a cancellation-free image under $\psi$ of $W^{\prime}=x_{1}^{-1} y^{-1} x_{1} x_{2} y x_{2}^{-1}$. Notice that in this process if $W$ is irredundant and orientable or nonorientable as a cyclic word, the same will be true of the ordinary word $W^{\prime}$.

For a quadratic word $W$, the set of all irredundant quadratic cyclic words with the genus and orientability of $W$, distinct up to automorphisms of $F$ that permute $X \cup X^{-1}$, is called the set of Wicks forms for $W$. M. Wicks showed in [19] that the orientable Wicks forms of genus one are $x^{-1} y^{-1} x y$ and $x^{-1} y^{-1} z^{-1} x y z$, and in [18] that the nonorientable Wicks forms of genus two are $x^{2} y^{2}, x y^{-1} x y, z^{-1} x^{2} z y^{2}$, and $x z x y z^{-1} y$. The nine maximal length orientable Wicks forms of genus two are listed by J. Comerford, L. Comerford, and Edmunds in [3]. A. Vdovina [written communication] has produced lists of the maximal length Wicks forms for nonorientable genus three and four and for orientable genus three.

Edmunds showed in [8] that if $Q \in F, Q$ is quadratic, $U \in H$, and $\phi$ is a solution to $Q=U$, then there is an endomorphism $\beta$ of $F$ such that $Q \beta$ is an irredundant quadratic word and $Q \beta=U$ has a cancellation-free solution. We modify Edmunds' proof to show that with $Q=\left[x_{1}, y_{1}\right] \ldots\left[x_{g}, y_{g}\right]$ or $Q=x_{1}^{2} \ldots x_{g}^{2}$ and our hypotheses on $U$, we may arrange to have $\beta$ an automorphism of $F$ and $\psi=\beta^{-1} \phi$. This will establish the conclusions of the theorem.

Proof of Theorem 1. We initially set $W=\left[x_{1}, y_{1}\right] \ldots\left[x_{g}, y_{g}\right]$ or $W=x_{1}^{2} \ldots x_{g}^{2}$ and $\psi=\phi$, and proceed by induction first on $N(W, \psi)=\sum_{x \in \operatorname{Var}(W)}|x \psi|$ and second on $|\operatorname{Var}(W)|$. We shall show that if $W, \psi$ do not satisfy the conclusions of the theorem, there is an automorphism $\beta$ of $F$ such that $W \beta$ is quadratic and either $N\left(W \beta, \beta^{-1} \psi\right)<N(W, \psi)$ or $N\left(W \beta, \beta^{-1} \psi\right)=N(W, \psi)$ and $|\operatorname{Var}(W \beta)|<|\operatorname{Var}(W)|$; we then replace $W$ by $W \beta$ and $\psi$ by $\beta^{-1} \psi$ and appeal to the induction hypothesis. Note that if $W, \psi$ do not satisfy the conclusions of the theorem, then either $W$ is redundant (which is not the case initially, but could 
occur later in the process), there are letters $x, y$ such that $x y$ is a subword of $W$ and there is cancellation in the product $(x \psi)(y \psi)$, or there is a variable $x$ in $W$ such that $x \psi=1$.

First, if $W$ is redundant, there are distinct letters $x$ and $y$ that occur in $W$ only in subwords $(x y)^{ \pm 1}$. We find that if $\beta: x \mapsto x y^{-1}, N\left(W \beta, \beta^{-1} \psi\right) \leq N(W, \psi)$ and $|\operatorname{Var}(W \beta)|<|\operatorname{Var}(W)|$. (When defining an endomorphism of $F$, we take it to fix all letters whose images are not specified.)

Suppose that there are letters $x$ and $y$ such that $x y$ is a subword of $W$ and there is cancellation in the product $(x \psi)(y \psi)$. Let $x \psi \equiv A B$ and $y \psi \equiv B^{-1} C$ with $B \neq 1$ and the product $A C$ freely reduced. In this case we define $\beta$ by $x \beta=x z$, $y \beta=z^{-1} y$ where $z$ is a variable that has not previously appeared in any quadratic word used in our process, and we specify that $z \psi=B$. There is no harm in this last requirement, since images under $\psi$ of variables not in $W$ are irrelevant to the value of $W \psi$. Here we see that $N\left(W \beta, \beta^{-1} \psi\right)<N(W, \psi)$.

Finally, suppose that $x \psi=1$ for some variable $x$ occurring in $W$. We must show that there is an automorphism $\beta$ of $F$ with $W \beta=W \tau$, where $\tau$ is the endomorphism of $F$ defined by $x \tau=1$. Note that our hypotheses ensure that, since $W \tau=U$ has a solution, genus ${ }^{+}(W \tau)=$ genus $^{+}(W)$ if $W$ is orientable, and that if $W$ is nonorientable, then $W \tau$ is nonorientable as well and genus ${ }^{-}(W \tau)=\operatorname{genus}^{-}(W)$. (We use the fact that if $W$ is a nonorientable quadratic word with genus ${ }^{-}(W)=g$ and if $V$ is an orientable quadratic word which is a homomorphic image of $W$, then genus ${ }^{+}(V) \leq(g-1) / 2$; cf. [13, Proposition I.6.10].) It follows that the initial and terminal vertices of the edge $e_{x}$ labeled by $x$ on $S_{W}$ are distinct. Let $v$ be the initial vertex of this edge. We define $\beta$ so that if $y$ is a variable other than $x$ with $e_{y}$ having initial but not terminal vertex at $v$ then $y \beta=x y$, so that if $y$ is a variable with $e_{y}$ having terminal but not initial vertex at $v$ then $y \beta=y x^{-1}$, and so that if $y$ is a variable with $e_{y}$ having both initial and terminal vertices at $v$ then $y \beta=x y x^{-1}$. Now $\beta$ is an automorphism, in fact a Whitehead automorphism, of $F$ and one may check that $W \beta=W \tau$. We see, then, that $N\left(W \beta, \beta^{-1} \psi\right) \leq N(W, \psi)$ and $|\operatorname{Var}(W \beta)|<|\operatorname{Var}(W)|$.

Theorem 11 shows us, then, that we can get representatives of all stabilizer classes of solutions to (11) and (2) by finding all the ways in which the right-hand side is a cancellation-free image of a Wicks form for the left-hand side. Note that the choice of $\gamma_{W}$ does not affect the stabilizer class, for if $\psi$ is a cancellation-free solution to $W=U$ with $W$ a Wicks form for $Q=\left[x_{1}, y_{1}\right] \ldots\left[x_{g}, y_{g}\right]$ or $Q=x_{1}^{2} \ldots x_{g}^{2}$, and if $\gamma_{1}$ and $\gamma_{2}$ are two automorphisms of $F$ sending $W$ to $Q$, then $\gamma_{1}^{-1} \gamma_{2} \in \operatorname{Stab}(Q)$ and so $\gamma_{1}^{-1} \psi$ and $\gamma_{2}^{-1} \psi$ are in the same stabilizer class of solutions to (1) or (2). Also note that if $W_{1}$ and $W_{2}$ are Wicks forms for $Q$ and $\psi_{1}$ and $\psi_{2}$ are cancellationfree solutions to $W_{1}=U$ and $W_{2}=U$ respectively and if there is a relabelling of variables $\rho$ (that is, an automorphism $\rho$ of $F$ that permutes $X \cup X^{-1}$ ) such that $W_{2}=W_{1} \rho$ and $\psi_{2}=\rho^{-1} \psi_{1}$, then for any maps $\gamma_{W_{1}}$ and $\gamma_{W_{2}}, \gamma_{W_{1}}^{-1} \psi_{1}$ and $\gamma_{W_{2}}^{-1} \psi_{2}$ are in the same stabilizer class of solutions to (11) or (2). Thus we find representatives of all stabilizer classes of solutions to (11) or (2) by finding all the ways the righthand side is a cancellation-free image of a Wicks form for the left-hand side, up to relabeling of variables in the Wicks form.

Now suppose that $W \in F$ is a Wicks form with $|W|=k$ and that $U \in H$ is cyclically reduced and $|U|=n$. The number of ways in which $U$ might be a cancellation-free image of $W$, as cyclic words, is bounded by the number of ways 
in which $U$ can be factored as a product of $k$ subwords, $\left(\begin{array}{c}n+k \\ k\end{array}\right)$, times the number of ways to make a first identification of a letter of $W$ with a chosen subword of $U$, which is $k$. Now $k\left(\begin{array}{c}n+k \\ k\end{array}\right)$ is a polynomial of degree $k$ in $n$ and, since every vertex on $S_{W}$ has degree at least three, we find that $k \leq 6(1-\chi(W))$. Further, the number of orientable or nonorientable Wicks forms of a given genus is finite. Thus we have the following, which is an instance of a more general result of Grigorchuck and Kurchanov [9, Theorem 4].

Corollary 1. Let $Q=\left[x_{1}, y_{1}\right] \ldots\left[x_{g}, y_{g}\right]$ or $Q=x_{1}^{2} \ldots x_{g}^{2}$ and let $U \in H$. Suppose that $U \in H^{\prime}$ and $\operatorname{genus}^{+}(U)=g$ if $Q=\left[x_{1}, y_{1}\right] \ldots\left[x_{g}, y_{g}\right]$, and that $U \in 2 H$ with genus $^{-}(U)=g$ and genus $^{+}(U) \geq g / 2$ if $Q=x_{1}^{2} \ldots x_{g}^{2}$. There is an algorithm to compute a complete set of representatives for stabilizer classes of solutions to $Q=U$ whose number of steps is bounded by a polynomial in $|U|$ of degree $12 g-6$ if $Q=\left[x_{1}, y_{1}\right] \ldots\left[x_{g}, y_{g}\right]$ and of degree $6 g-6$ if $Q=x_{1}^{2} \ldots x_{g}^{2}$ and $g>1$.

\section{Applications}

We use our classification of solutions to sharpen a theorem of R. Burns, Edmunds, and I. Farouqi [2, Theorem 1], which in turn improved upon a result of Ju. Hmelevskii [10]. All of this is based on pioneering work of A. I. Mal'cev 14.

Theorem 2. Suppose that $U \in H$ is nontrivial and cyclically reduced and that there are solutions to

$$
[x, y]=U
$$

Then every solution to (4) is in the stabilizer class of a solution $\phi_{0}$ with $\left|x \phi_{0}\right| \leq$ $(1 / 2)|U|,\left|y \phi_{0}\right| \leq(1 / 2)|U|$, and $\left|x \phi_{0}\right|+\left|y \phi_{0}\right| \leq|U|-1$. Further, $U$ has a cyclic permutation $U^{*}$ such that every solution to $[x, y]=U^{*}$ is in the stabilizer class of a solution $\phi_{0}^{*}$ with $\left|x \phi_{0}^{*}\right| \leq(1 / 2)|U|-1,\left|y \phi_{0}^{*}\right| \leq(1 / 2)|U|-1$, and $\left|x \phi_{0}^{*}\right|+\left|y \phi_{0}^{*}\right| \leq$ $(2 / 3)|U|$.

Proof of Theorem . Given the orientable Wicks forms of genus one, we know that as a cyclic word $U$ factors as either $X^{-1} Y^{-1} X Y$ or $X^{-1} Y^{-1} Z^{-1} X Y Z$ with $X, Y$, $Z$ nonempty. As an ordinary word, then, we find that up to change of notation either

$$
U \equiv X_{1}^{-1} Y^{-1} X_{1} X_{2} Y X_{2}^{-1}
$$

or

$$
U \equiv X_{1}^{-1} Y^{-1} Z^{-1} X_{1} X_{2} Y Z X_{2}^{-1}
$$

where $X_{1} X_{2}, Y$, and $Z$ are nonempty, although one of $X_{1}$ or $X_{2}$ may be empty. If (5) holds, $U=\left[X_{2} X_{1}, X_{1}^{-1} Y X_{2}^{-1}\right]$ gives the first conclusion of Theorem 2, and $U^{*}=X_{2}^{-1} X_{1}^{-1} Y^{-1} X_{1} X_{2} Y=\left[X_{1} X_{2}, Y\right]$ gives the second. If (6) obtains, $U=$ $\left[X_{2} Y X_{1}, X_{1}^{-1} Z X_{2}^{-1}\right]$ gives the first assertion of the theorem. To see the second set of inequalities, we let $U^{*}=X_{2}^{-1} X_{1}^{-1} Y^{-1} Z^{-1} X_{1} X_{2} Y Z$ and note that $2\left|X_{1} X_{2}\right|+$ $2|Y|+2|Z|=|U|$ implies that one of $\left|X_{1} X_{2}\right|,|Y|,|Z|$ is less than or equal to $(1 / 6)|U|$. If $\left|X_{1} X_{2}\right| \leq(1 / 6)|U|$, we write $U^{*}=\left[Y X_{1} X_{2}, X_{2}^{-1} X_{1}^{-1} Z\right]$; if $|Y| \leq(1 / 6)|U|$, we write $U^{*}=\left[Y X_{1} X_{2}, Y Z\right]$; if $|Z| \leq(1 / 6)|U|$, we write $U^{*}=\left[Z^{-1} X_{1} X_{2}, Y Z\right]$. In each case, we see the truth of the second assertion of Theorem 2 .

Theorem 1 of 2] does not include the hypothesis that $U$ is cyclically reduced, and asserts that every solution to (4) is in the stabilizer class of a solution $\phi_{0}$ with 
$\left|x \phi_{0}\right| \leq|U|-3$ and $\left|y \phi_{0}\right| \leq|U|-3$. We lose no generality in assuming that $U$ is cyclically reduced, for equations $[x, y]=C^{-1} U_{0} C$ and $\left[C x C^{-1}, C y C^{-1}\right]=U_{0}$ have the same solutions.

The bounds given in Theorem 2 for $|x \phi|+|y \phi|$ are not tight in the case that $|U|=4$, for then every solution is in the stabilizer class of a solution $\phi_{0}$ of (4) with $\left|x \phi_{0}\right|=\left|y \phi_{0}\right|=1$. For $|U|>4$, though, we shall show that the bounds given in Theorem 2 do not admit improvement.

Let $n$ be a positive integer and let $\left\{a_{1}, \ldots, a_{n}, b_{1}, \ldots, b_{n}, c_{1}, \ldots, c_{n}\right\}$ be a subset of $A$, our chosen set of free generators for $H$. Consider first

$$
U_{1}=b_{n}^{-1} \ldots b_{1}^{-1} c_{1}^{-1} b_{1} \ldots b_{n} a_{1} \ldots a_{n} c_{1} a_{n}^{-1} \ldots a_{1}^{-1} .
$$

We find that, cyclically, $U_{1}$ is not a cancellation-free image of $x^{-1} y^{-1} z^{-1} x y z$, and that $U_{1}$ is a cancellation-free image of $x^{-1} y^{-1} x y$ in only one way, up to change of variables. Thus every solution to $[x, y]=U_{1}$ is in the stabilizer class of $\phi_{0}$, where $x \phi_{0}=a_{1} \ldots a_{n} b_{1} \ldots b_{n}$ and $y \phi_{0}=a_{1} \ldots a_{n} c_{1} a_{n}^{-1} \ldots a_{1}^{-1}$. Now elements of $\operatorname{Stab}([x, y])$ are automorphisms of $\langle x, y ;\rangle$, so for any solution $\phi$ to (雨), $\{x \phi, y \phi\}$ and $\left\{x \phi_{0}, y \phi_{0}\right\}$ generate the same subgroup of $H$. Since $\left\{x \phi_{0}, y \phi_{0}\right\}$ is Nielsen reduced, it follows that for any solution $\phi$ to $[x, y]=U_{1},|x \phi|+|y \phi| \geq\left|x \phi_{0}\right|+\left|y \phi_{0}\right|=\left|U_{1}\right|-1$.

Now let

$$
U_{2}=a_{n}^{-1} \ldots a_{1}^{-1} b_{n}^{-1} \ldots b_{1}^{-1} c_{n}^{-1} \ldots c_{1}^{-1} a_{1} \ldots a_{n} b_{1} \ldots b_{n} c_{1} \ldots c_{n} .
$$

A typical cyclic permutation of $U_{2}$ is

$$
U_{2}^{*}=a_{i}^{-1} \ldots a_{1}^{-1} b_{n}^{-1} \ldots b_{1}^{-1} c_{n}^{-1} \ldots c_{1}^{-1} a_{1} \ldots a_{n} b_{1} \ldots b_{n} c_{1} \ldots c_{n} a_{n}^{-1} \ldots a_{i+1}^{-1}
$$

with $0 \leq i \leq n$. As above, $[x, y]=U_{2}^{*}$ has only one stabilizer class of solutions, namely that of $\phi_{0}^{*}$ given by

$$
\begin{aligned}
x \phi_{0}^{*} & =a_{i+1} \ldots a_{n} b_{1} \ldots b_{n} a_{1} \ldots a_{i}, \\
y \phi_{0}^{*} & =a_{i}^{-1} \ldots a_{1}^{-1} c_{1} \ldots c_{n} a_{n}^{-1} \ldots a_{i+1}^{-1} .
\end{aligned}
$$

Again we find that $\left\{x \phi_{0}^{*}, y \phi_{0}^{*}\right\}$ is Nielsen reduced, so for any solution $\phi^{*}$ to $[x, y]=$ $U_{2}^{*},\left|x \phi^{*}\right|+\left|y \phi^{*}\right| \geq\left|x \phi_{0}^{*}\right|+\left|y \phi_{0}^{*}\right|=(2 / 3)\left|U_{2}\right|$.

By similar arguments, we can see that for any cyclic permutation $U_{1}^{*}$ of $U_{1}$ and solution $\phi^{*}$ of $[x, y]=U_{1}^{*}$, either $\left|x \phi^{*}\right| \geq(1 / 2)\left|U_{1}\right|-1$ or $\left|y \phi^{*}\right| \geq(1 / 2)|U|-1$.

Our next result shows that an equation of the form $[x, y]=T$ with $T \in H$ may have any number of stabilizer classes of solutions.

Theorem 3. Suppose that $H=K * L$ with $K$ and $L$ free, that $U \in K$ and $V \in L$ are nontrivial and not proper powers, and that $m$ and $n$ are positive integers. The equation

$$
[x, y]=\left[U^{m}, V^{n}\right]
$$

has $m+n-1$ distinct stabilizer classes of solutions represented by $x \phi_{i}=U^{m}$, $y \phi_{i}=U^{-i} V^{n}$ for $0 \leq i<m$ and by $x \psi_{j}=V^{j} U^{m}, y \psi_{j}=V^{n}$ for $1 \leq j<n$.

Proof. We note that if $\alpha$ is an automorphism of $H$, solutions $\mu$ and $\nu$ of (7) are in the same stabilizer class if and only if $\mu \alpha$ and $\nu \alpha$ are in the same stabilizer class of solutions to $[x, y]=\left[(U \alpha)^{m},(V \alpha)^{n}\right]$. Applying an automorphism of $H$ induced by inner automorphisms of $K$ and $L$, then, we may assume that $U$ and $V$ are cyclically reduced.

We next need to find the ways in which $\left[U^{m}, V^{n}\right]$, as a cyclic word, can be factored as $X^{-1} Y^{-1} X Y$ or $X^{-1} Y^{-1} Z^{-1} X Y Z$. In any such factorization, $X^{ \pm 1}$, 
$Y^{ \pm 1}$, and $Z^{ \pm 1}$ must be subwords of $U^{ \pm m}$ and $V^{ \pm n}$. If, for example, $X^{-1}$ overlapped both factors of the product $U^{-m} V^{-n}$, then $X$ would overlap both factors of either $V^{-n} U^{m}$ or $V^{n} U^{-m}$, but this would violate the cyclic reduction of either $V$ or $U$. If $\left[U^{m}, V^{n}\right]=X^{-1} Y^{-1} X Y$ as cyclic words, then, it must be that up to change of notation $X=U^{m}$ and $Y=V^{n}$; this gives solution $\phi_{0}$. If $\left[U^{m}, V^{n}\right]=X^{-1} Y^{-1} Z^{-1} X Y Z$ as cyclic words, one of $X, Y, Z$ must be $U^{ \pm m}$ or $V^{ \pm n}$. If, say, $Z=V^{n}$, then $X Y=U^{m}$ and $X^{-1} Y^{-1}=U^{-m}, X$ and $Y$ commute, and so $X, Y$, and $U$ are powers of a common element. Since $U$ is not a proper power, $X=U^{i}$ and $Y=U^{m-i}$ for some $i, 1 \leq i<m$. This gives us the solution $x \mapsto Z^{-1} X=V^{-n} U^{i}, y \mapsto Y Z=U^{m-i} V^{m}$, which is in the stabilizer class of $\phi_{i}$. Likewise if, say, $X=U^{m}$, then $Y=V^{j}$ and $Z=V^{n-j}$ for some $j, 1 \leq j<n$, which gives rise to the solution $x \mapsto Z^{-1} X=V^{-n+j} U^{m}, y \mapsto Y Z=V^{n}$, in the stabilizer class of $\psi_{j}$.

It remains to show that $\phi_{0}, \phi_{1}, \ldots, \phi_{m-1}, \psi_{1}, \ldots, \psi_{n-1}$ are in different stabilizer classes. We do this by proving that the subgroups $S_{i}, T_{j}$ of $H$ generated by $\left\{x \phi_{i}, y \phi_{i}\right\}$ for $0 \leq i<m$ and by $\left\{x \psi_{j}, y \psi_{j}\right\}$ for $1 \leq j<n$ are all different.

We first show that if $0 \leq i<j<m$, then $S_{i} \neq S_{j}$. We do this by cases. First, if $\left|V^{n}\right| \leq\left|U^{i}\right|$ and $\left|V^{n}\right| \leq\left|U^{m-i}\right|,\left\{U^{-i} V^{n}, U^{m-i} V^{n}\right\}$ is a Nielsen reduced generating set for $S_{i}$ and we see that $U^{m-j} V^{n} \in S_{j}-S_{i}$. Next, if $i \leq m / 2$ and $\left|V^{n}\right|>\left|U^{i}\right|$ or $\left|V^{n}\right|>\left|U^{m-i}\right|$, then $\left|V^{n}\right|>\left|U^{i}\right|$ and $\left\{U^{-i} V^{n}, U^{m}\right\}$ is a Nielsen reduced generating set for $S_{i}$. We again note that $U^{m-j} V^{n} \in S_{j}-S_{i}$. Finally, if $i>m / 2$ and either $\left|V^{n}\right|>\left|U^{i}\right|$ or $\left|V^{n}\right|>\left|U^{m-i}\right|$, then $\left|V^{n}\right|>\left|U^{m-i}\right|$ and $\left\{U^{m-i} V^{n}, U^{m}\right\}$ is a Nielsen reduced generating set for $S_{i}$, and once more $U^{m-j} V^{n} \in S_{j}-S_{i}$.

In a similar way, we show that for $1 \leq i<j<n, T_{i} \neq T_{j}$. Finally, we note that $U^{m}$ is a member of each of the $S_{i}$ but none of the $T_{j}$, which distinguishes the $S_{i}$ from the $T_{j}$.

Theorem 4. If $a_{1}, \ldots a_{g}$ are distinct free generators of a free group $H$ and if $n_{1}, \ldots n_{g}$ are nonzero integers, every solution to

$$
x_{1}^{2} \ldots x_{g}^{2}=a_{1}^{2 n_{1}} \ldots a_{g}^{2 n_{g}}
$$

is in the stabilizer class of the solution $\phi_{0}$ given by $x_{i} \phi_{0}=a_{i}^{n_{i}}$ for $1 \leq i \leq g$.

Proof. Let $U=a_{1}^{2 n_{1}} \ldots a_{g}^{2 n_{g}}$. It is clear that $U \notin H^{\prime}$, that $U \in 2 H$, and that genus $^{-}(U) \leq g$. Suppose that genus ${ }^{-}(U)=k$ and let $\phi$ be a solution to $x_{1}^{2} \ldots x_{k}^{2}=$ $U$. By Theorem 1, there is an irredundant nonorientable quadratic $W \in F=$ $\left\langle x_{1}, \ldots ;\right\rangle$ with genus ${ }^{-}(W)=k$ and a cancellation-free solution $\psi$ to $W=U$ such that if $\gamma_{W}$ is an automorphism of $F$ with $W \gamma_{W}=x_{1}^{2} \ldots x_{k}^{2}$, then $\phi=\sigma \gamma_{W}^{-1} \psi$ for some $\sigma \in \operatorname{Stab}\left(x_{1}^{2} \ldots x_{k}^{2}\right)$.

There is no variable $y$ such that both $y$ and $y^{-1}$ occur in $W$, for there is no $Y \in H, Y \neq 1$, such that both $Y$ and $Y^{-1}$ are (cyclically) subwords of $U$. Further, the form of $U$ shows that for each letter $y$ in $W, y \psi$ is contained in a single syllable $a_{i}^{2 n_{i}}$ of $U$. Thus $W \equiv W_{1} \ldots W_{g}$ with $W_{1}, \ldots W_{g}$ words on disjoint sets of variables and with $W_{i} \psi=a_{i}^{2 n_{i}}$ for $1 \leq i \leq g$. It follows that each $W_{i}$ is nonorientable quadratic with genus ${ }^{-}\left(W_{i}\right) \geq 1$, so $k=$ genus $^{-}(W)=\sum_{i=1}^{g} \operatorname{genus}^{-}\left(W_{i}\right) \geq g$. Thus genus ${ }^{-}(W)=g$ and genus ${ }^{-}\left(W_{i}\right)=1$ for $1 \leq i \leq g$, so each $W_{i}$ is the square of a variable or its inverse. We may take $\gamma_{W}$ to be an automorphism of $F$ that permutes $\left\{x_{1}, x_{1}^{-1}, \ldots\right\}$, and so $\gamma_{W}^{-1} \psi=\phi_{0}$ and $\phi$ is in the stabilizer class of $\phi_{0}$. 


\section{Commutators as Products of Squares}

It is well known that in a free group, indeed in any group, every commutator is a product of three squares:

$$
[U, V]=\left(U^{-1}\right)^{2}\left(U V^{-1}\right)^{2} V^{2} .
$$

One cannot in general get by with fewer than three squares; for example, Lyndon and Morris Newman [12 have shown that in the free group on $a$ and $b,[a, b]$ is not a product of two squares. We can see that this is true, for $[a, b]$ is not a cancellation-free image of any of the nonorientable Wicks forms of genus two.

In a free group, a nontrivial commutator is never a square; this was first noted by M. Schützenberger [17], and follows from examination of the orientable Wicks forms of genus one. Thus one is left with the question of which commutators are products of two squares in a free group. One obvious possibility is that $[U, V]=\left[S^{2}, T\right]$ for some $S$ and $T$, in which case $[U, V]=\left(S^{-1}\right)^{2}\left(T^{-1} S T\right)^{2}$. This includes the situation that $[U, V]=\left[P, Q^{2}\right]$ for some $P$ and $Q$, for $\left[P, Q^{2}\right]=\left[P^{-1} Q^{2} P, P^{-1}\right]$. One might ask if this is the only way in which a commutator can be a product of two squares in a free group. We shall give an example to show that this is not the case. This complements an example given by J. Comerford and Y. Lee [4] to show that if in a free group a product of two commutators is a square, it need not be the square of a commutator.

Our example fits into a sequence of results about solutions to equations of the form

$$
x_{1}^{2} \ldots x_{g}^{2}=1
$$

in a free group. It is easy to see that if $g=1$, (9) implies that $x_{1}=1$, and that if $g=2$, (9) implies that $x_{2}=x_{1}^{-1}$. For $g=3$, Lyndon proved [11] that (9) implies that $x_{1}, x_{2}$, and $x_{3}$ are powers of a common element. Thus for $g \leq 3$, (9) has only "obvious" solutions. When $g=5$, (9) can be rewritten using an automorphism of $\left\langle x_{1}, \ldots, x_{5} ;\right\rangle$ as $x_{1}^{2}=\left[x_{2}, x_{3}\right]\left[x_{4}, x_{5}\right]$; J. Comerford and Lee showed $[4$ that this has "nonobvious" solutions. The case we consider here is $g=4$, in which (9) can be rewritten as $x_{1}^{2} x_{2}^{2}=\left[x_{3}, x_{4}\right]$. Again, we show that this has "nonobvious" solutions.

We now give our example. Let $U=b^{-1} a^{-1} b^{2} a b^{-1}$ and $V=a$ in $H=\langle a, b ;\rangle$. One can check that

$$
[U, V]=\left(b a^{-1} b^{-1} a^{-1} b^{-1} a b a b^{-1}\right)^{2}\left(b a^{-1} b^{-1} a^{-1} b^{2} a b^{-1} a\right)^{2},
$$

but we shall show that $\left[x^{2}, y\right]=[U, V]$ has no solutions in $H$. This follows from the following two results.

Lemma 1. Every solution to $[x, y]=[U, V]$ is in the stabilizer class of $x \phi_{0}=U$, $y \phi_{0}=V$.

Lemma 2. If $K$ is the subgroup of $H$ generated by $U$ and $V$, if $W \in K$, and if $x^{2}=W$ has a solution in $H$, then $x^{2}=W$ has a solution in $K$.

To see that these lemmas imply that $\left[x^{2}, y\right]=[U, V]$ has no solutions, suppose that $\psi$ is a solution to $\left[x^{2}, y\right]=[U, V]$. Let $x \psi=P$ and $y \psi=Q$. By Lemma $\mathbb{1}$, $P^{2}=x \sigma \phi_{0}$ and $Q=y \sigma \phi_{0}$ for some $\sigma \in \operatorname{Stab}([x, y])$. Thus $P^{2}$ and $Q$ are elements of $K$ and, by Lemma 2, $P$ is an element of $K$. Now $U$ and $V$ are free generators for $K$, so $\beta: K \rightarrow\langle x, y$; $\rangle$ defined by $U \beta=x$ and $V \beta=y$ is an isomorphism. It follows that $\beta \sigma \phi_{0}$ is an automorphism of $K$ that sends $U$ to $P^{2}$, so $U$ is the square of an element of $K$, which is impossible. 
Proof of Lemma Q . We must show that as a cyclic word,

$$
[U, V]=b a^{-1} b^{-2} a b a^{-1} b^{-1} a^{-1} b^{2} a b^{-1} a
$$

does not factor as $X^{-1} Y^{-1} Z^{-1} X Y Z$ and factors as $X^{-1} Y^{-1} X Y$ in essentially only one way.

Suppose first that there is a factorization of the cyclic word $[U, V]$ in which the copy of $a b$ consisting of the last and first letters of the ordinary word $[U, V]$ is contained in one of $X^{ \pm 1}, Y^{ \pm 1}, Z^{ \pm 1}$, say in $X$. Then $X \equiv X_{1} a b X_{2}$ with $X_{1}$ and $X_{2}$ possibly empty, and since $b^{-1} a^{-1}$ occurs in $[U, V]$ in only one position,

$$
a^{-1} b^{-2} a b a^{-1}=X_{2} Y X_{2}^{-1} \text { or } X_{2} Y Z X_{2}^{-1}
$$

and

$$
b^{2} a b^{-1}=X_{1}^{-1} Y^{-1} X_{1} \text { or } X_{1}^{-1} Y^{-1} Z^{-1} X_{1} .
$$

But these imply that $a^{-1} b^{-2} a b a^{-1}$ is conjugate to $\left(b^{2} a b^{-1}\right)^{-1}$, which is not the case.

It must be, then, that up to change of notation,

$$
b a^{-1} b^{-2} a b a^{-1} b^{-1} a^{-1} b^{2} a b^{-1} a=X^{-1} Y^{-1} X Y \text { or } X^{-1} Y^{-1} Z^{-1} X Y Z .
$$

By a length comparison,

$$
b a b^{-2} a b a^{-1}=X^{-1} Y^{-1} \text { or } X^{-1} Y^{-1} Z^{-1}
$$

and

$$
b^{-1} a^{-1} b^{2} a b^{-1} a=X Y \text { or } X Y Z .
$$

Thus $X$ begins and ends with $b^{-1}$. The possibilities are $X=b^{-1} a^{-1} b^{2} a b^{-1}=U$ and $Y=a=V$ or $X=b^{-1}$. But $X=b^{-1}$ implies that $a^{-1} b^{2} a b^{-1} a$ is conjugate to $\left(a b^{-2} a b a^{-1}\right)^{-1}$, which isn't so.

Proof of Lemma 粵. Let us suppose that $W \neq 1$. Since $\{U, V\}$ is a free generating set for $K$, there is a unique expression $W=Z(U, V)$ with $Z$ a freely reduced word in $U$ and $V$. In fact, the map $x \mapsto U, y \mapsto V$ is a cancellation-free solution to $Z(x, y)=W$. We may assume without loss of generality that $Z(U, V)$ is a cyclically reduced word on $U$ and $V$, and hence that $W$ is a cyclically reduced word on $a$ and $b$.

Now suppose that $W=T^{2}$ for some (cyclically reduced) $T \in H$. If $T \notin K$, then $T \equiv T_{0} U_{1}$ with $T_{0} \in K$ and $U=U_{1} U_{2}$ a nontrivial factorization of $U=$ $b^{-1} a^{-1} b^{2} a b^{-1}$. But this implies that some nontrivial initial subword $U_{1}$ of $U$ is an element of $K$, which is plainly not the case. Thus $T \in K$ and $W$ is a square in $K$.

We close with a brief description of how we found this example. Using generators for the nonorientable mapping class groups given by J. Birman and D. Chillingworth [1], J. Comerford and Lee [4] provided generating sets for the stabilizers of nonorientable quadratic words. They gave a set of five generators, $\bar{a}_{1}, \bar{b}_{1}, \bar{c}_{1}, \bar{b}_{2}$, and $y_{3}$, for the stabilizer of $W=s_{1} t_{1} s_{1}^{-1} t_{1}^{-1} s_{2} t_{2} s_{2}^{-1} t_{2}$. Now if $\alpha$ is the automorphism of $\left\langle s_{1}, t_{1}, s_{2}, t_{2} ;\right\rangle$ that sends $t_{2}$ to $s_{2} t_{2}$ and fixes $s_{1}, t_{1}$, and $s_{2}$, we see that $W \alpha=s_{1} t_{1} s_{1}^{-1} t_{1}^{-1} s_{2}^{2} t_{2}^{2}$, and that $\sigma \in \operatorname{Stab}(W)$ if and only if $\alpha^{-1} \sigma \alpha \in \operatorname{Stab}(W \alpha)$. The idea, then, is to start with the "obvious" solution $\phi_{0}$ to $W \alpha=1$ given by $s_{1} \phi_{0}=1, t_{1} \phi_{0}=a, s_{2} \phi_{0}=b^{-1}, t_{2} \phi_{0}=b$, and preceed it with elements of $\operatorname{Stab}(W \alpha)$ to produce "nonobvious" solutions to $W \alpha=1$. The example presented here is based on the solution $\alpha^{-1} y_{3}^{2} \alpha \phi_{0}$ to $W \alpha=1$. 


\section{REFERENCES}

[1] J. S. Birman and D. R. J. Chillingworth, On the homeotopy group of a non-orientable surface, Math. Proc. Cambridge Philos. Soc. 71 (1972), 437-448.

[2] R. G. Burns, C. C. Edmunds, and I. H. Farouqi, On commutator equalities and stabilizers in free groups, Canad. Math. Bull. 19 (1976), no. 3, 263-267.

[3] J. A. Comerford, L. P. Comerford, Jr., and C. C. Edmunds, Powers as products of commutators, Comm. Algebra 19 (1991), no. 2, 675-684.

[4] J. A. Comerford and Y. Lee, Product of two commutators as a square in a free group, Canad. Math. Bull. 33 (1990), no. 2, 190-196.

[5] L. P. Comerford, Jr. and C. C. Edmunds, Genus of powers in a free group, To appear.

[6] Solutions of equations in free groups in Group Theory: Proceedings of the 1987 Singapore Conference, Walter de Gruyter, 1989, pp. 347-356.

[7] M. Culler, Using surfaces to solve equations in free groups, Topology 20 (1981), 133-145.

[8] C. C. Edmunds, On the endomorphism problem for free groups II, Proc. London Math. Soc. (3) 38 (1979), 153-168.

[9] R. I. Grigorchuk and P. F. Kurchanov, On quadratic equations in free groups, Comtemporary Math. 131 (1992), 159-171.

[10] Ju. I. Hmelevskiı̌, Systems of equations in a free group, I, Math. USSR Izvestija 5 (1971), no. $6,1245-1276$.

[11] R. C. Lyndon, The equation $a^{2} b^{2}=c^{2}$ in free groups, Michigan Math. J. 6 (1959), 89-95.

[12] R. C. Lyndon and Morris Newman, Commutators as products of squares, Proc. Amer. Math. Soc. 39 (1973), no. 2, 267-272.

[13] R. C. Lyndon and P. E. Schupp, Combinatorial group theory, Springer-Verlag, 1977.

[14] A. I. Mal'cev, On the equation $z x y x^{-1} y^{-1} z^{-1}=a b a^{-1} b^{-1}$ in a free group, Algebra i Logika 1 (1962), no. 5, 45-50 (Russian).

[15] W. S. Massey, Algebraic topology: An introduction, Springer-Verlag, 1977.

[16] G. Rosenberger, Minimal generating systems for plane discontinuous groups and an equation in free groups in Proceedings of Groups-Korea 1988, Springer-Verlag, 1989, pp. 170-186.

[17] M. P. Schützenberger, Sur l'equation $a^{2+n}=b^{2+m} c^{2+p}$ dans un groupe libre, C. R. Acad. Sci. Paris Sér. I Math. 248 (1959), 2435-2436.

[18] M. J. Wicks, The equation $x^{2} y^{2}=g$ over free products in Proceedings of the Second Congress of the Singapore National Academy of Science, 1973, pp. 238-248.

[19] M. J. Wicks, Commutators in free products, J. London Math. Soc. 37 (1962), 433-444.

Department of Mathematics, Eastern Illinois University, Charleston, Illinois 61920, U. S. A.

E-mail address: cflpc@eiu.edu

Department of Mathematics, Mount Saint Vincent University, Halifax, Nova Scotia B3M 2J6, CANADA

E-mail address: CEDMUNDS@linden.msvu.ca 